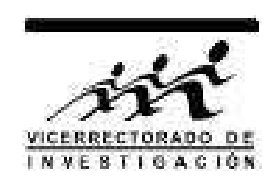

\title{
Fuente Sísmica del Terremoto de Chile 2010 a partir de Inversión de Datos y Observaciones Geodésicas
}

\author{
César Jiménez ${ }^{* 1}$, Miguel Saavedra ${ }^{1}$, Nabilt Moggiano ${ }^{2}$ y Nick Moreno ${ }^{1}$ \\ ${ }^{1}$ Universidad Nacional Mayor de San Marcos, Ap. Postal 14-0149, Lima, Perú \\ ${ }^{2}$ Universidad Continental, Huancayo - Perú
}

Recibido 05 noviembre 2017 - Aceptado 28 enero 2018

\begin{abstract}
El 27 de febrero de 2010 un terremoto de magnitud 8.8 Mw (según el USGS) sacudió la región centro-sur de Chile con un saldo de más de 500 muertos. Como efecto cosísmico se originó un maremoto que destruyó muchos poblados y balnearios costeros, así como la deformación permanente de la corteza. Esta deformación cosísmica puede ser cuantificada mediante mediciones geodésicas: GPS, observaciones de campo en el litoral y datos de interferometría satelital InSAR. Del análisis y procesamiento de los datos geodésicos se puede obtener los parámetros que caracterizan la distribución de la fuente sísmica mediante un proceso de inversión, en el cual se compara la data simulada con la data observada a través de un proceso de inversión con el método de mínimos cuadrados no-negativos. Los resultados muestran la existencia de dos asperezas principales situados al norte y al sur del epicentro. El valor máximo de la dislocación es $17.3 \mathrm{~m}$ ubicado en la parte norte del área de ruptura. El momento "geodésico" escalar calculado es $1.25 \times 10^{22} \mathrm{Nm}$, que equivale a una magnitud de $8.7 \mathrm{Mw}$.
\end{abstract}

Palabras claves: Terremoto, deformación cortical, inversión geodésica.

\section{Seismic Source of the Chile 2010 Earthquake from Inversion of Geodetic Data and Ob- servations}

On February 27, 2010 an earthquake of magnitude 8.8 Mw (according to USGS) shook the center-southern region of Chile with a balance of more than 500 deaths. As a coseismic effect, a tsunami was generated which destroyed many coastal villages, as well as the permanent crustal deformation. This coseismic deformation can be quantified by geodetic measurements: GPS, field observations at the littoral and InSAR satellite interferometry data. From the analysis and processing of the geodetic data it is possible to obtain the parameters that characterize the distribution of the seismic source through an inversion process, in which the simulated data is compared with the observed data using the non-negative least squares method. The results show the existence of two main asperities located to the north and south of the epicenter. The maximum slip or dislocation was $17.3 \mathrm{~m}$ located in the northern side of the rupture area. The calculated "geodetic"scalar moment is $1.25 \times 10^{22} \mathrm{Nm}$, which is equivalent to a magnitude of $8.7 \mathrm{Mw}$.

Keywords: Earthquake, crustal deformaction, geodetic inversion.

\section{Introducción}

La Tierra no es un cuerpo estático, sino que ha estado y está sujeta a una continua actividad, es decir, la Tierra es un sistema dinámico. Esto se debe a la interacción de las placas tectónicas que generan esfuerzos geológicos al interior de la tierra. La corteza terrestre está constituida por los casquetes tectónicos, los cuales, en su interacción, producen esfuerzos y deformaciones.

La deformación de la corteza terrestre puede ser cuan- tificada mediante observaciones geodésicas como: datos GPS (Global Posittioning System), observaciones de campo en el litoral e imágenes satelitales de interferometría radar (InSAR). Estas observaciones proporcionan la deformación cosísmica vertical (levantamiento o subsidencia) en la ubicación de las estaciones o puntos de observación que serán utilizados en el modelo de inversión geodésica para obtener la distribución de la fuente sísmica (sin considerar la evolución temporal de la fuente), luego a partir de esto se obtiene el campo (y los vectores) de deformación

"cjimenezt@unmsm.edu.pe 
para la zona de estudio.

Desde un punto de vista físico, la inversión de datos o señales geodésicas proporciona mejores resultados que la inversión de ondas telesísmicas, para obtener la distribución de la fuente sísmica. Esto se debe a que el modelo de fuente sísmica debe ajustarse a los datos geodésicos observados, sin considerar un modelo de velocidad de propagación de ondas sísmicas. En el segundo caso (inversión de ondas telesísmicas), el modelo de estructura de velocidades no se conoce muy bien, se asume un modelo de velocidad que proporcione la mejor correlación durante el proceso de inversión, además el modelo sísmico, por lo general, subestima la amplitud de la deformación cosísmica.

\section{Antecedentes de investigaciones previas}

Muchos investigadores han estudiado los aspectos sismológicos, geodésicos y mareográficos del evento de Chile 2010.

Moreno et al., (2012) usaron la inversión de datos GPS para obtener la distribución de la fuente sísmica. Los desplazamientos cosísmicos estáticos fueron obtenidos de 47 estaciones no-permanentes (SGPS) y 8 estaciones continuas (CGPS) alrededor del terremoto de Maule [1].

Vigny et al., (2011) usaron datos de estaciones geodésicas GPS para obtener la distribución de la fuente sísmica mediante inversión, la máxima dislocación (slip) fue de $15 \mathrm{~m}$, también obtuvieron el patrón de deformación post-sísmica [2].

Lorito et al., (2011) usaron datos de tsunami y datos geodésicos (observaciones de cambios del nivel del mar, InSAR y 6 estaciones GPS) para obtener la distribución de la dislocación (slip) y los cambios de esfuerzos cosísmicos inducidos mediante un proceso de inversión [3].

Fujii et al., (2012) usaron datos de tsunami y datos geodésicos (observaciones de cambios del nivel del mar: levantamiento o subsidencia) para obtener la distribución del slip mediante un proceso de inversión [4].

En la presente investigación se ha obtenido la distribución de la fuente sísmica y el campo de deformación cosísmica a partir de la inversión de datos geodésicos GPS, observaciones de campo y datos de interferometría satelital InSAR, mediante el método de los mínimos cuadrados no negativos.

\section{Sismicidad en la región central de Chile}

El terremoto de Chile de 2010 se generó en los límites entre la placa tectónica de Nazca y la de Sudamérica. Estas dos placas convergen a una razón de $80 \mathrm{~mm}$ por año. El sismo es del tipo de falla inversa en la interfase entre las dos placas, con la placa de Nazca moviéndose por debajo de la placa Sudamericana, proceso conocido como subducción. La actividad sísmica dominante a lo largo de la costa de Chile es una consecuencia directa del proceso de subducción de la placa de Nazca bajo la placa Sudamericana, por lo que la costa de Chile tiene una historia de grandes terremotos. En los últimos cinco siglos (para los cuales se cuenta con información histórica) se han producido varios terremotos destructores de magnitud mayor a 8.0 Mw en diversos lugares del territorio chileno (Madariaga, 1998) [5].

Las zonas de gap sísmico más importantes en Chile son: la del norte que coincide con la zona de ruptura del terremoto de 1877 entre Arica y Antofagasta, y la segunda entre Concepción y Constitución en el centro-sur del país, donde ningún gran terremoto de subducción se ha producido en esta zona desde el terremoto de Concepción en 1835 hasta el terremoto del 2010.

Debido al terremoto de Maule de 2010, murieron 708 personas y desaparecieron 19 personas, hubo un gran daño en la infraestructura de edificios, casas, carreteras y puentes. Este terremoto (de magnitud $8.8 \mathrm{Mw}$ ) es el más fuerte en Chile después del gran terremoto de 1960 de magnitud 9.5 Mw, el mayor terremoto registrado instrumentalmente [6].

Como efecto cosísmico se produjo la generación de un tsunami o maremoto (con efectos devastadores para muchas localidades costeras cercanas a la zona de ruptura) y la subsidencia así como el levantamiento cosísmico (uplift) de la placa tectónica continental, según los datos geodésicos de estaciones GPS permanentes, que en algunos lugares fue hasta de $2 \mathrm{~m}$ [2].

\section{Datos}

\section{Parámetros Hipocentrales}

Según los datos del reporte final del National Earthquake Information Center (USGS, https://earthquake.usgs.gov/earthquakes/map), los parámetros hipocentrales son:

Fecha: 27 Feb 2010

Hora GMT: 06:34:11.5

Latitud: $-36.12^{\circ}$

Longitud: $-72.90^{\circ}$

Profundidad: $22 \mathrm{~km}$

Magnitud: $8.8 \mathrm{Mw}$

\section{Datos Geodésicos}

Los datos geodésicos permiten cuantificar los desplazamientos cosísmicos y la deformación de la corteza en las 3 dimensiones espaciales: Norte, Este y Vertical. Para ello, se utilizaron los siguientes tipos de datos geodésicos:

1) Observaciones de campo del levantamiento o subsidencia cosísmica en zonas costeras, que proporcionan sólo la componente vertical de la deformación (Farías et al, 2010 [7], Fritz et al., 2011 [8]). 
2) Los datos de Interferometría Satelital InSAR, también proporcionan sólo la componente vertical de la deformación, los datos son accesibles desde:

$\mathrm{ftp}$ ://topex.ucsd.edu/pub/chile_eq/chile_insar.zip

3) Datos de deformación cosísmica (para 3 componentes: Norte, Este y Vertical) de estaciones GPS permanentes y de campaña (Vigny et al, 2011) [2].

La Figura 1 muestra un mapa con la ubicación de las estaciones geodésicas GPS (en azul), los lugares donde se realizó medidas de campo (en amarillo) y los puntos donde se realizó el cálculo con datos de interferometría satelital InSAR (en rojo).

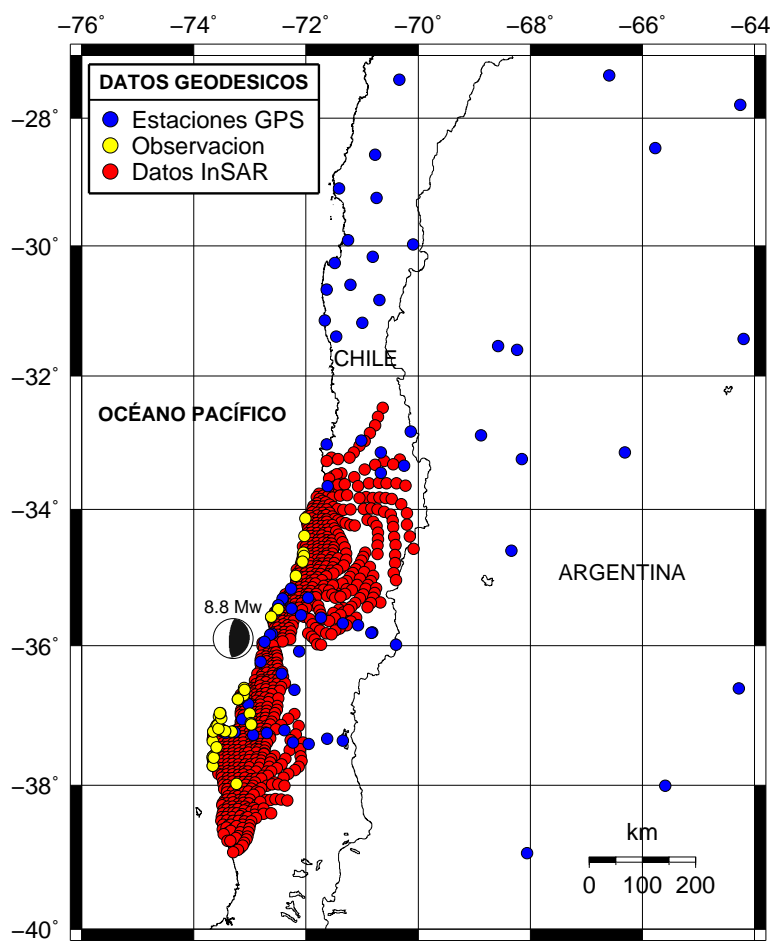

Figura 1: Mapa de ubicación de las estaciones geodésicas GPS (círculos azules), datos InSAR (círculos rojos) y observaciones de campo (círculos amarillos).

\section{Área de ruptura a partir de las réplicas}

Las réplicas ocurridas dentro de los primeros días, permiten evaluar la extensión del área de ruptura de la fuente sísmica.

Se ha contabilizado un total de 374 eventos de magnitud mayor a $4.0 \mathrm{Mw}$, desde la ocurrencia del terremoto principal (27 Feb 2010) hasta un día (24 horas) después. La réplica más fuerte ocurrió el 02 de enero de 2011 y tuvo una magnitud de $7.1 \mathrm{Mw}$ (lat $=-73.35^{\circ}$, lon=-38.37 $)$.

Estas réplicas están distribuidas principalmente entre la fosa y la línea de costa y en menor proporción en el continente a todo lo largo de la geometría de ruptura desde Valparaíso hasta la región del Bio-bio, entre la latitud $-33^{\circ}$ hasta la latitud $-39^{\circ}$ cubriendo una superficie con longitud de alrededor de $500 \mathrm{~km}$, teniendo un ancho de alrededor de 150 km (Figura 2).

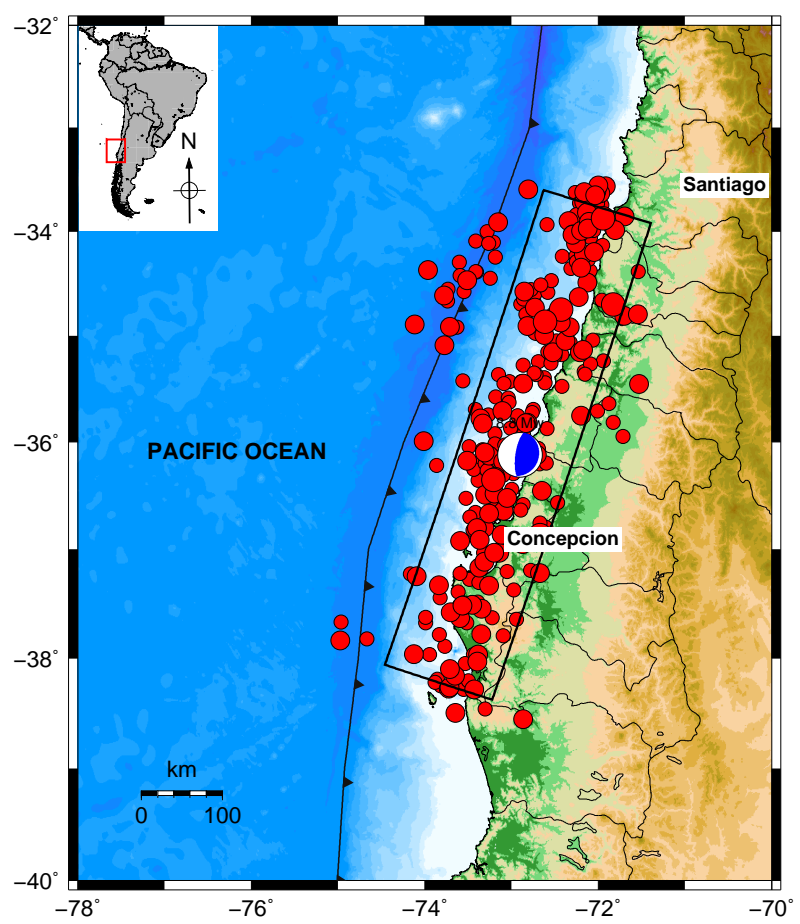

Figura 2: Mapa de ubicación de las réplicas (en color rojo) ocurridos dentro de las 24 primeras horas. El rectángulo negro representa el área de ruptura.

\section{Metodología}

La metodología para obtener los resultados de este estudio, se lista a continuación:

1. Adquisición de los datos geodésicos: GPS, InSAR y observaciones de campo.

2. Delimitación del área de estudio de acuerdo a la distribución geográfica de la data geodésica: GPS, InSAR y observación de campo (levantamiento y subsidencia).

3. Obtención del mecanismo focal a partir del Global CMT (http://www.globalcmt.org/) u otra fuente.

4. Determinación de la geometría de ruptura (a partir de la distribución de las réplicas) y de los parámetros de falla.

5. Cálculo de la deformación elástica según la formulación de Okada (1992) [9].

6. Interpolación de la deformación elástica en la posición de las estaciones y/o mediciones geodésicas (interp_def.m). 
7. Obtención de la matriz de Green $G$ y obtención del vector de datos $d$.

8. Modelo de inversión: $G m=d$. Inversión por el método de mínimos cuadrados no negativos.

\section{Método de Inversión}

Si se conocen los parámetros que caracterizan a un sistema y sus condiciones iniciales ( $y$ de frontera) entonces se puede predecir el comportamiento de dicho sistema en cualquier instante de tiempo. A este tipo de problema, de predecir el comportamiento futuro, se le llama el "problema directo". El "problema inverso" consiste en utilizar los resultados de ciertas observaciones para inferir los valores de los parámetros que caracterizan al sistema (Tarantola, 2002) [10]. En este trabajo se utilizaron los registros mareográficos del maremoto para deducir los parámetros de la fuente sísmica (distribución del slip).

El proceso será considerado como un "Sistema Lineal e Invariante en el Tiempo" que posee dos propiedades importantes: a) linealidad, que permite utilizar el principio de superposición para las funciones de Green y para las deformaciones unitarias y $b$ ) invariancia en el tiempo, que permite realizar un desplazamiento en el tiempo de las señales observadas y sus correspondientes funciones de Green, sincronizando las señales observadas y simuladas.

La solución del problema inverso no es única, sino que se elige el conjunto de valores que proporcionen la mejor correlación entre los datos observados y simulados.

\section{Parámetros de la fuente}

Para estimar la distribución de la dislocación o slip se utilizó el método de inversión de los datos geodésicos de calidad aceptable. El área de ruptura se dividió en 40 fragmentos iguales con dimensiones: $L=52 \mathrm{~km}$ y $W=30 \mathrm{~km}$, ubicados a una profundidad $H$ con respecto a la parte superior (según la coordenada vertical) de cada subfalla, numerados de sur a norte.

$$
\begin{aligned}
H_{j} & =h+\frac{W}{2} \operatorname{sen} \delta[1-\bmod (j, 2)], \quad j=1, \ldots, 8 \\
h & =h_{e}-\left[\left(x_{e}-x_{0}\right) \cos \theta-\left(y_{e}-y_{0}\right) \sin \theta\right] \tan \delta
\end{aligned}
$$

donde, $h$ es la profundidad de la parte superior del área de ruptura, $\left(x_{0}, y_{0}\right)$ son las coordenadas de la esquina inferior izquierda, $j$ representa la posición de la $j$-ésima subfuente, $\delta$ es el ángulo de buzamiento, $\bmod (j, 2)$ representa el módulo o resto de la división de $j$ con respecto a 2, $\left(x_{e}, y_{e}, h_{e}\right)$ son las coordenadas del hipocentro o foco sísmico y $\theta$ es el ángulo azimutal. Se debe notar que $H_{j}$ no es igual a la profundidad focal, pero están relacionados a través de la Ec.(2).

La deformación cosísmica se calcula para cada subfalla con una dislocación o slip unitario, utilizando la formulación de Okada (1992) y los datos del mecanismo focal.
Estos parámetros del mecanismo focal son tomados del Global CMT (http://www.globalcmt.org/) y son iguales para cada una de los fragmentos (subfuentes):

Angulo azimutal $=19^{\circ}$ (strike angle)

Angulo de buzamiento $=18^{\circ}$ (dip angle)

Angulo de dislocación $=116^{\circ}$ (rake angle)

\section{Cálculo de las funciones de Green}

Desde el punto de vista físico, una función de Green representa la respuesta del sistema, señal simulada en la $i$ ésima estación, cuando dicho sistema es perturbado por un impulso unitario, una deformación debido a la dislocación unitaria en la $j$-ésima sub-fuente. Usando este campo de deformación como condición inicial, se calculan las formas de onda o funciones de Green en cada estación simulando la propagación del maremoto por el método de diferencias finitas para cada una de las sub-fuentes, usando una batimetría real. Las formas de onda mareográficas observadas son expresadas como una combinación lineal de las formas de ondas calculadas, en notación tensorial como,

$$
G_{i j}(t) m_{j}=d_{i}(t)
$$

donde $G_{i j}$ es la función de Green en la $i$-ésima estación generada por la $j$-ésima subfuente, $m_{j}$ es el valor del slip en la $j$-ésima subfuente y $d_{i}$ es la deformación observada en la $i$-ésima estación geodésica.

\section{Inversión}

La inversión se realizó mediante el método de mínimos cuadrados no-negativos [11]. Dicho método consiste en comparar la señal simulada con la señal observada y hacer mínimo el cuadrado del residual,

$$
\left|G_{i j} m_{j}-d_{i}\right|^{2} \rightarrow 0
$$

con la condición: $m_{j}>0$. Este algoritmo garantiza que todos los valores de la dislocación (slip) sean positivos, como una condición necesaria desde el punto de vista físico. Sin embargo, matemáticamente este modelo no será el resultado más óptimo con respecto al método de mínimos cuadrados simple. El resultado de la inversión se muestra en la Figura 3 


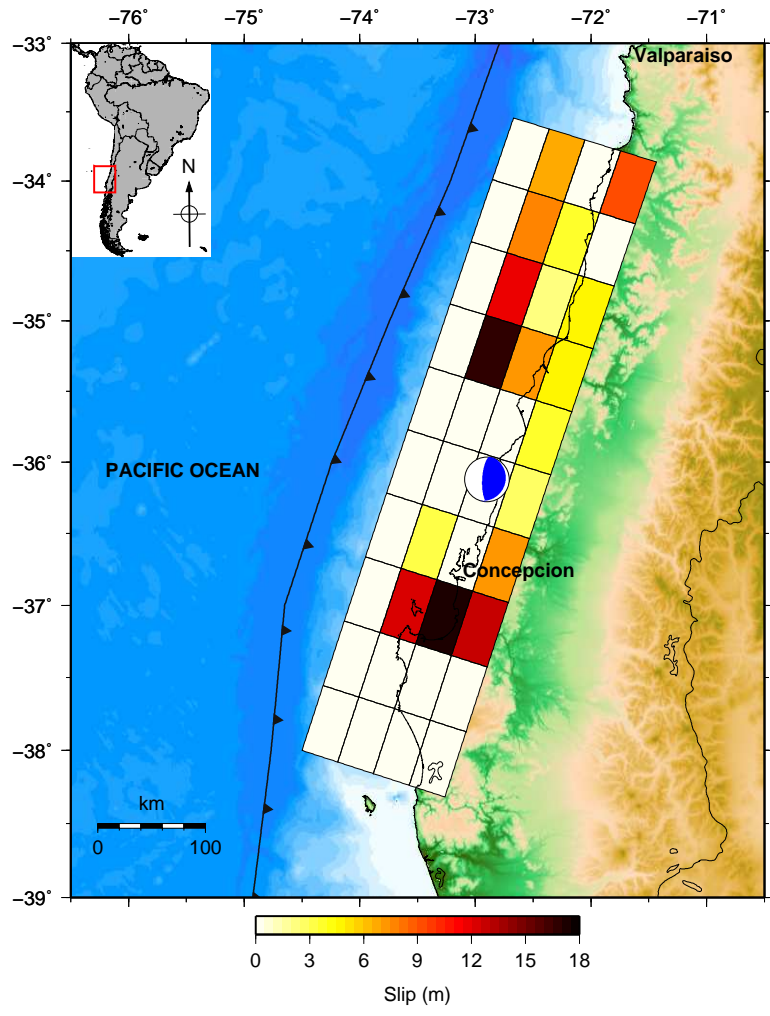

Figura 3: Distribución de la dislocación o fuente sísmica del terremoto de Chile 2010.

\section{Resultados y discusión}

A partir de los valores del slip se obtiene el campo de deformación total como una combinación lineal de las deformaciones unitarias, lo cual será utilizado como condición inicial de la propagación del maremoto. El máximo valor del slip es de $17.3 \mathrm{~m}$ y se ubica en la parte norte del área de ruptura, frente a la ciudad de Constitución. Esto explica el hecho de que la ciudad de Constitución (latitud $-35.32^{\circ}$ ) haya sido la que más daño ha sufrido a causa de la inundación por tsunami, puesto que se encuentra en la vecindad de la zona de mayor liberación de energía. El máximo valor de la deformación cosísmica inicial es de 6.39 $\mathrm{m}$.

Debido a la posición del epicentro y a la geometría del área de ruptura, se puede inferir que el proceso de ruptura se propagó en forma bidireccional: desde el epicentro (latitud $-36.12^{\circ}$ ) hacia el norte donde se concentra la mayor deformación y hacia el sur con una menor deformación (Figura 4). Esto concuerda con los resultados obtenidos por otros métodos como inversión telesísmica [12] e inversión mareográfica [4].

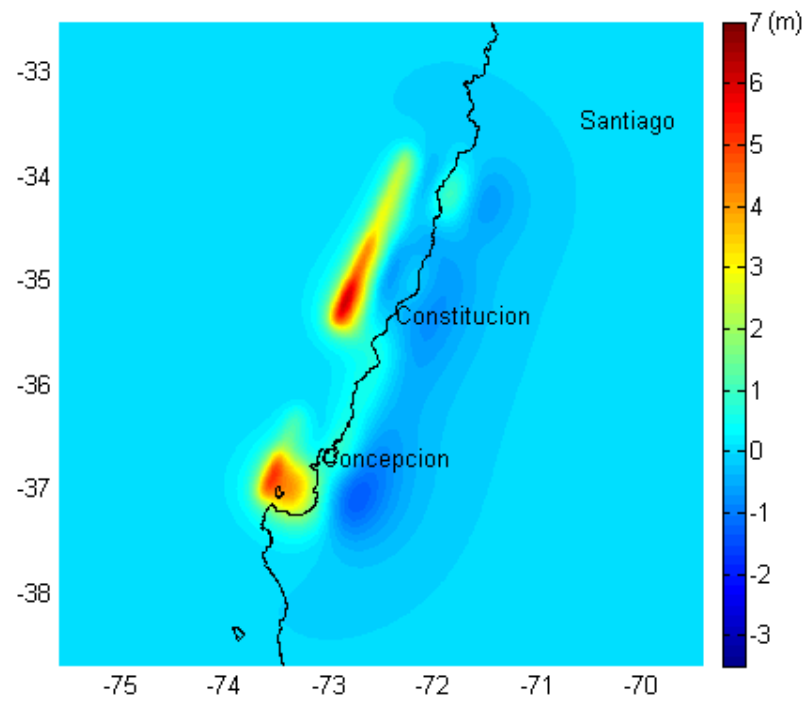

Figura 4: Distribución de la componente vertical de la deformación cosísmica para el terremoto de Chile 2010.

\section{Momento geodésico escalar}

Teniendo la distribución de la dislocación o slip y las dimensiones de cada subfuente es posible calcular el momento geodésico escalar que se define como $M_{0}=\mu L W D$, donde $M_{0}$ es el momento sísmico, $\mu=6.0 \times 10^{10} \mathrm{~N} / \mathrm{m}^{2}$ es la rigidez promedio del medio elástico, $L=52 \mathrm{~km}$ es la longitud de cada subfuente, $W=30 \mathrm{~km}$ es el ancho de cada subfuente y $D=3.35 \mathrm{~m}$, es el slip promedio. El momento sísmico escalar total es $M_{0}=1.25 \times 10^{22} \mathrm{Nm}$, y según la relación $M_{w}=\frac{2}{3} \log \left(M_{0}\right)-6.07$, se obtiene una magnitud en la escala de momento de $8.7 \mathrm{Mw}$. Lo que significa que a partir de los datos geodésicos se obtiene un valor similar a la magnitud Mw calculada a partir de los registros sísmicos.

\section{Conclusiones}

La aspereza principal (zona de mayor liberación de energía sísmica) tuvo una dislocación o slip de $17.3 \mathrm{~m}$ y se ubica en la parte norte del área de ruptura, frente a la ciudad de Constitución-Chile (latitud $-35.33^{\circ}$, longitud $-72.41^{\circ}$ ) (Figura 4).

Se considera que el proceso de ruptura tuvo una directividad bidireccional a lo largo del azimut, siendo el epicentro el punto de ruptura inicial, esta se propagó hacia el sur y hacia el norte, en forma simultánea.

Considerando un coeficiente de rigidez elástica promedio de $\mu=6.0 \times 10^{10} \mathrm{~N} / \mathrm{m}^{2}$, el momento sísmico escalar calculado es de $M_{0}=1.25 \times 10^{22} \mathrm{Nm}$ y la magnitud del terremoto inferido a partir de los datos geodésicos da un valor de $8.7 \mathrm{Mw}$. 
La máxima amplitud de la deformación cosísmica vertical para este modelo es de $6.39 \mathrm{~m}$. Los datos y los resultados de esta investigación muestran que muchas zonas costeras sufrieron un levantamiento cosísmico.

Matemáticamente, el problema inverso tiene muchas soluciones. Fisicamente, se elige la solución que proporcione la mejor correlación entre los datos observados y simulados. Una forma de validar el modelo de fuente sísmica obtenido es mediante la comparación de los datos geodésicos observados y simulados.

\section{Referencias}

[1] M. Moreno, D. Melnick, M. Rosenau, J. Baez, J. Klotz, O. Oncken, A. Tassara, J. Chen, K. Bataille, M. Bevis, A. Socquet, J. Bolte, C. Vigny, B. Brooks, I. Ryder, V. Grund, B. Smalley, D. Carrizo, M. Bartsch, and $\mathrm{H}$. Hase. Toward understanding tectonic control on the Mw 8.82010 Maule Chile earthquake. Earth and Planetary Science Letters 321-322, pp 152-165. (2012).

[2] C. Vigny, A. Socquet, S. Peyrat, J. Ruegg, M. Métois, R. Madariaga, S. Morvan, M. Lancieri, M. Lacassin, J. Campos, D. Carrizo, M. Bejar, S. Barrientos, R. Armijo, C. Aranda, M. Valderas, I. Ortega, F. Bondoux, S. Baize, H. Lyon-Caen, A. Pavez, J. Vilotte, M. Bevis, B. Brooks, R. Smalley, H. Parra, J. Baez, M. Blanco, S. Cimbaro, E. Kendrick. The $2010 \mathrm{Mw}$ 8.8 Maule megathrust earthquake of Central Chile, Monitored by GPS. Science, vol 332, pp 1417-1421. (2011).

[3] S. Lorito, F. Romano, S. Atzori, X. Tong, A. Avallone, J. McCloskey, M. Cocco, E. Boschi and A. Piatanesi. Limited overlap between the seismic gap and coseismic slip of the great 2010 Chile earthquake. Nature Geosciences, doi: 10.1038/NGEO1073. (2011).
[4] Y. Fujii, y K. Satake. Slip Distribution and Seismic Moment of the 2010 and 1960 Chilean Earthquakes Inferred from Tsunami Waveforms and Coastal Geodetic Data. Pure Appl. Geophys., doi: 10.1007/s00024-012-0524-2. (2012).

[5] H. Madariaga. Sismicidad de Chile. Física de la Tierra, No. 10, pp 221 - 258. Universidad Complutense de Madrid. (1998).

[6] H. Kanamori. The Energy Release in Great Earthquakes. JGR, vol 82, No. 20, pp 2981-2987. (1977).

[7] M. Farias, G. Vargas, A. Tassara, S. Carretier, S. Baize, D. Melnick, K. Bataille. Land level changes produced by the Mw 8.82010 Chilean Earthquake. Science-xpress, vol 329, pp 916 (2010).

[8] H. Fritz, C. Petroff, P. Catalán R. Cienfuegos, P. Winckler, N. Kalligeris, R. Weiss, S. Barrientos, G. Meneses, C. Valderas, C. Ebeling. A. Papadopoulus, M. Contreras, R. Almar, J. Dominguez and C. Synolakis. Field Survey of the 27 February 2010 Chile Tsunami. PAAG, vol 168, pp 1989-2010. (2011).

[9] Y. Okada. Internal deformation due to shear and tensile faults in a half space. BSSA, vol 82, No. 2, pp 1018-1040. (1992).

[10] A. Tarantola. Inverse problem theory and methods for model parameter estimation. Ed. Society for Industrial and Applied Mathematics. Philadelfia. (2002).

[11] C. Lawson and R. Hanson. Solving Least Squares Problems. Prentice-Hall, (1974).

[12] N. Pulido, Y. Yagi, H. Kumagai and M. Nishimura. Rupture process and coseismic deformations of the 2010 Maule Earthquake, Chile. Earth Planets Space, vol 63, pp 955-959 (2011). 